\title{
APPLICATION OF ACTOR-CRITIC REINFORCEMENT LEARNING METHOD FOR CONROL OF A SAGITTAL ARM DURING OSCILLATORY MOVEMENT
}

\author{
Vahid Golkhou', Caro Lucas ${ }^{2}$, Mohamad Parnianpour' \\ ${ }^{1}$ Department of Mechanical Engineering. Biomechanics Lab, Sharif University of Technology, \\ Tehran, Iran \\ ${ }^{2}$ Department of Electrical and Computational Engineering, University of Tehran, Tehran, Iran
}

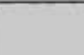

ABSTRACT

Numerous disciplines are engaged in studies involving motor control. In this study, we have used a single link system with a pair of muscles that are excited with alpha and gamma signals to achieve an oscillatory movement with variable amplitude and frequency.

The system is highly nonlinear in all its physical and physiological attributes. The major physiological characteristics of this system are simultaneous activation of a pair of nonlinear muscle-like-actuators for control purposes, existence of nonlinear spindle-like sensors and Golgi tendon organ-like sensor, actions of gravity and external loading. Transmission delays are included in the afferent and efferent neural paths to account for a more accurate representation of the reflex loops. The profile of excitation is difficult to predict a priori, hence we have used a reinforcement learning approach to track a desired trajectory.

This paper proposes a reinforcement learning method with an Actor-Critic architecture instead of middle and low level of central nervous system (CNS). The Actor in this structure is a two layer feedforward neural network and the Critic is a model of the cerebellum. The Critic is trained by State-Action-Reward-State-Action (SARSA) method. The Critic will train the Actor by supervisory learning based on previous experiences. The system is implemented on a PC using Matlab ${ }^{\circledR}$ and Simulink $^{\text {*x }}$ Software. To enhance the computational performance a number of $C$ codes were also written.

The effectiveness and the biological plausibility of the present model are demonstrated by several simulations.

The system showed excellent tracking capability and after 280 epochs the RMS error for position and velocity profiles were $0.02,0.04$ radian and radian/sec, respectively:
\end{abstract}

Biomed Eng Appl Basis Comm, 2004 (December); 16: 305-312.

Keywords: motor control, reinforcement learning, Actor-Critic, CMAC, Simulink

\section{INTRODUCTION}

Received: Apr 5, 2004; Accepted: Oct 10, 2004

Comespondence: $M$. Pamianpour, Professor

Depariment of Mechanical Engineering. Sharif University of Technology, Azadi Ave., Tehran, Iran. P.O. Box $11365-9567$

E-majl: pamianpour@sharif.edu
One of the most important and poorly understood functions of the central nervous system in humans is the control of movement. An understanding of the central nervous system as it pertains to locomotion is important to scientists who interpret kinesiological events related to the coordination of the body, and to bioengineers who design prosthetic, orthotic, and neuromuscular simulation systems in order to resiore 
impaired or lost neuromotor units.

In biomechanics, neurosciences and rehabilitation engineering, a fundamental goal is to derive appropriate sets of neuromuscular excitation signals for specific human movements and maneuvers. The inverse dynamics analysis is a commonly used technique whereby kinematic measurements are used as inputs to solve for the actuator control signals. The required joint torques to execute a specified movement can be calculated from the dynamic equations of motion [1-2], or derived from neural networks that learn the inverse dynamics of the system [3-4]. Once the joint torques are computed, it is desirable to determine the force contribution of each muscle to the net joint torque in order to compute the desired neural excitation signals.

The problem, however, arises in many living systems where several pairs of muscles span the same joint and contribute to the same joint torque. The individual muscle forces, producing the net joint torque cannot be uniquely determined due to the redundancy in the system. Various cost functions and optimization procedures are frequently used to distribute the net joint torque, at each instant of time, into muscle forces.

Such cost functions may not be physiologically justified. Also, there may be little correlation between computed and measured muscular forces [5] because the ground reaction forces, accelerations, and other forces of contact with the environment may also be involved in the generation of forces by the muscles [6].

Various techniques ranging from state feedback to optimal control have been proposed to derive the needed muscle forces for specific maneuvers [5, 7-8].

However, robust general algorithms for solving such high dimensional complex nonlinear control problems are far from being well-developed. Also, proposed techniques, in many cases, have physiological limitations. For example, inverse optimization methods, which are extensively used in biomechanics, efficiently article changes in muscle force generation but provide a limited viewpoint with regard to how external and tissue loading conditions cause changes in task strategy. Kinematics variation and postural stability are not explicitly addressed within most of the inverse optimization modeling frameworks [8]. In addition, many musculoskeletal motion studies [4, 9-10] do not address the issue of physiological compatibility and alpha and gamma inputs as excitation signals.

Recently, reinforcement learning has attracted attention as a learning method for studying movement planning [9, 11-13]. Houk et al. [14] have proposed a hypothesis that the basal ganglia might involve a type of reinforcement learning method known as ActorCritic learning. Moreover, some researchers believe that reinforcement learning is essential for understanding human movements [15]. It is difficult, however, to apply reinforcement learning to the system with any hidden state of the environment, that is, with non-Markov property [10]. Biological control systems include the long time delay (at least $100 \mathrm{~ms}$ ) associated with neural transmission and neural computation, which represents a non-Markov property.

In this paper, we present an Actor-Critic Controller in which the Actor is a neural network linkage system and the Critic is a network that represents a model of the cerebellum. This architecture is used to control a single link musculoskeletal system in sagittal plane. The performance and capability of the proposed controller are demonstrated by simulating various oscillatory movements.

\section{THE MUSCLE MODEL}

A modified version of Hill based muscle model developed by Kim et al. [16] was used. The actuator has two neural inputs, a force output and two sensory outputs.

The equation of muscle-like-actuator is modified in the way that the passive elastic term generates the force only when the current length of the contractile element is longer than the initial length of the contractile element. The modified equation of the actuator force is as follows:

$$
\begin{gathered}
F_{c}=\left(R+c \Delta l_{c} u\left(\Delta_{c}\right)\right)^{2}+b \Delta\left(l_{c}\right) u\left(-l_{c}\right) \\
\Delta l_{c}=l_{c}-l_{c o}
\end{gathered}
$$

Where the subscript $c$ refers to the contractile element, $l_{c}$ is the current length of the contractile element, $l_{c o}$ is the resting length of the contractile element and $\Delta l_{c}$ is the difference in length from its resting length. The variable $R$ is the firing rate input, $b$ is the viscous constant of the contractile element and $c$ is the passive elastic constant. $u(x)$ refers to the unit step function.

In addition to the firing rate inputs issued from the alpha motoneurons, the neuromuscular system has the gamma neural input to the muscle [17]. The gamma neural input comes directly from the mid-level of the CNS and is modeled as a threshold signal in the spindle organ.

The contractile element is followed by a low-pass filter $H(s)$ and a linear spring that jointly represent the action of the tendon [18]. The equations for the tendon are 


$$
\begin{aligned}
& F_{t}=H(s) F_{c} \\
& F_{t}=\beta \Delta l_{t}
\end{aligned}
$$

Where subscript $t$ refers to tendon, $\Delta l_{t}$ is the excursion of the tendon from its resting length, and $\beta$ is the stiffness constant of the tendon, which is very large $(>=40000[\mathrm{~N} / \mathrm{m}])[19]$.

\section{SENSOR DYNAMIC}

The neuromuscular control system has two sensors: a muscle spindle organ and a Golgi tendon organ. One of two spindle types is the primary intrafusal fiber that is sensitive to velocity as well as length where as secondary is only sensitive to length. The spindle model implemented was proposed by Gielen and Houk [20] and modified by Kim and Hemami [16] to provide an appropriate sensory signal:

$$
\begin{aligned}
& R_{\mathrm{s} p}=s_{1}\left(l_{c}-T h\right) u\left(l_{c}-T h\right)+ \\
& s_{2}\left(l_{c}-T h\right) u\left(l_{c}-T h\right) l_{c}^{\tau} u\left(l_{c}\right)
\end{aligned}
$$

Where $R_{s p}$ is the spindle response, $s_{1}$ and $s_{2}$ are positive gain constants, $l_{c}$ is the contractile element length and $\tau$ is a positive fraction.

In this paper, Th is the threshold length, and gamma input that is assumed to be the desired contractile element length at each instant.

The spindle response signal is added to alpha signal of the same muscle and is subtracted from the alpha signal the other (antagonist) muscle.

It is also assumed that the spindle signal has a transmission delay $\Delta$ in its reflex path to the spinal cord $[16,21]$.

The Golgi tendon organ is sensitive to tension [17]. We used the GTO model that is proposed by Kim and Hemami [16].

$$
R_{g t t}=z\left(F-F_{t h}\right) u\left(F-F_{t h}\right) F^{\prime \prime} u(F)
$$

Where $R_{g t o}$ is the Golgi tendon organ response, $z$ is positive gain constant, $F$ is the actuator force, $F_{t h}$ is the threshold force and $v$ is a positive fraction. It is assumed that the GTO signal has the same transmission delay $\Delta$ in its reflex path. Because GTO signal inhibits the firing rate of the motoneurons, it is subtracted from the excitation signal of the agonist muscle. Note, the transmission delay in reflex loops by spindles and GTO sensors is important because it has crucial effect on generating undesirable tremor.

\section{THE MODEL OF PHYSICAL SYSTEM}

The system consists of a planar one-link arm hinged to an inertial reference frame and acted upon by two muscle-like-actuators: an extensor that rotates the arm clockwise and a flexor that rotates the arm counterclockwise (Fig. 1). The two states of the system are the angle $\theta$ measured from the vertical and the angular velocity $\dot{\theta}$.

The physical parameters of the musculoskeletal system are its mass $m$, the distance of the center of gravity from the hinge $d$, the moment of inertia of the arm about the center of elbow joint $J$, the moment arms of the flexor and the extensor which are assumed to be equal to each other and constant, $k$. No friction or damping is assumed at the joint (Fig. 1). Let $F_{e}$ and $F_{f}$ be the extensor and the flexor forces, respectively. Let the constant of gravity be $g$. The differential equation of the system is given by:

$$
J \theta-m g d \sin (\theta)=k\left(F_{e}-F_{f}\right)
$$

The block diagram of the musculoskeletal system is depicted in Fig. 2.

\section{THE CONTROL PROBLEM}

The main aim of this control problem is to learn how to generate the appropriate neural (alpha and gamma) signals in order to track a desired trajectory that may be designed by the higher level controller at the CNS.

Hence, we used the reinforcement learning concept that is based on trial and error methodology and constant evaluation of performance [22].

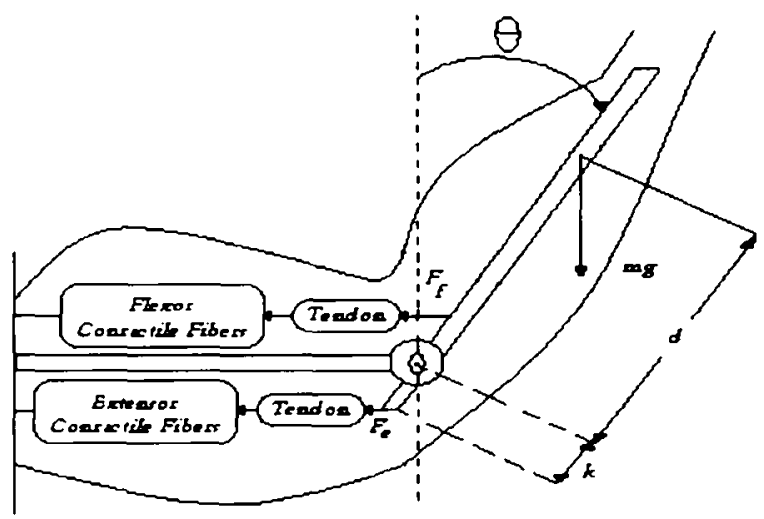

Fig. 1 The Musculoskeletal System. 


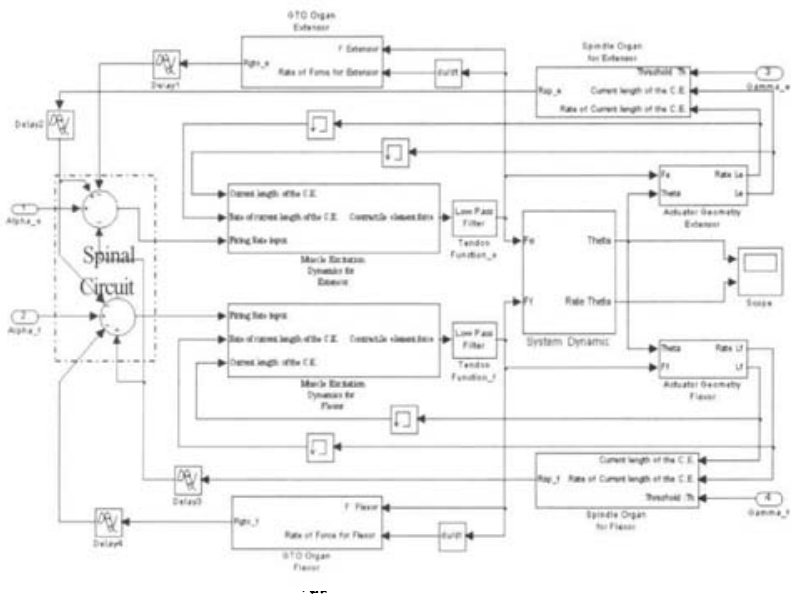

Fig. 2 Block diagram of proposed musculoskeletal system. The diagram consists of skeletal system, spinal circuit, muscle excitation dynamic, tendon, spindle organ sensor, GTO sensor, delay in reflex loops, all for both extensor and flexor muscles.

When we think about the nature of learning, the idea that we learn by interacting with our environment is perhaps the first that comes to mind. When an infant plays, waves her arms, or looks about, she has no explicit teacher, but she does have a direct sensorimotor connection to her environment. Exercising this connection produces a wealth of information about cause and effect, about the consequences of actions, and about what to do in order to achieve goals. Throughout our lives, such interactions are undoubtedly a major source of knowledge about our environment and ourselves [22].

We applied a reinforcement learning method based on the Actor-Critic architecture.

Actor-Critic methods are the natural extension of the idea of reinforcement comparison methods to Temporal Difference (TD) learning [23]. The Actor network can be thought of as the control agent, because it implements a policy. The Actor network is part of the dynamic system as it interacts directly with the system by providing control signals for the plant. The Critic network implements the reinforcement learning part of the control system as it provides policy evaluation and can be used to perform policy improvement. This learning agent architecture has the advantage of implementing both a reinforcement learning mechanism as well as a control mechanism [24]. Because the policy is computed explicitly in the Actor network, we can meet the real-time demands of the control system [24]. For the Actor, we selected the two-layer, feedforward network with tanh hidden units and linear output units.

$$
\begin{gathered}
\mu_{j}=\sum_{i=1}^{n} W_{i, j} e_{i} \\
u_{k}=\sum_{j=1}^{h} V_{k, j} \tanh \left(\mu_{j}\right)
\end{gathered}
$$

For the Critic, since we know from biology that cerebellum holds an internal feedforward model for control movements [25], we propose the CMAC (Cerebellar Model Articulation Controller) network [26-27]. In this paper, we also applied the Critic, CMAC, to estimate the optimal action to update the weights in the actor network that performs the control task. The critic is trained to produce the expected sum of future reinforcement that will be observed given the current state and action. The Critic is trained using State-Action-Reward-State-Action (SARSA) method which is a variant of reinforcement learning developed by Sutton [28].

CMAC is a class of sparse coarse-coded memory that models Cerebellar functionality [26]. Each input or state-action pair activates a specific set of memory locations or features, the arithmetic sum of whose contents is the value of the stored Q-value. The CMAC takes advantage of the continuous nature of the input and is able to store the necessary data in a physical memory of practical size. A CMAC consists of several overlapping tilings of the state-action space to produce the feature representation. A query is performed by first activating all the features that contain the stateaction input and then summing the values of all the activated features. CMACs have been widely used in conjunction with reinforcement learning [28-29]. The size of a CMAC depends on the number of tilings and the size of each tiling. The implemented algorithm is highlighted in Fig. 3.

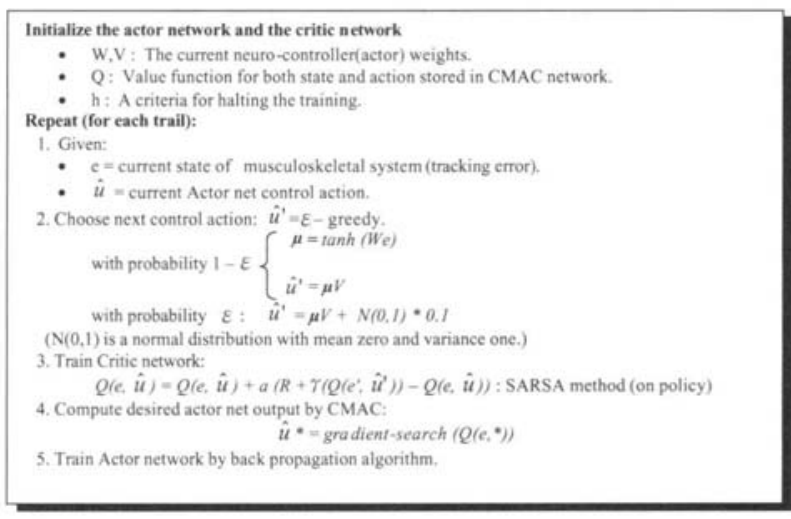

Fig.3 Learning algorithm used by the critic, CMAC, using State-Action-Reward-State-Action (SARSA) method. 
The Actor network receives the tracking error from the musculoskeletal system to produce a control signal. It is trained via back propagation (gradient descent) algorithm and training example provided by Critic net.

In this work, the reinforcement signal was simply defined to be the magnitude of the error between the reference signal (produced by high level controller of CNS) and the musculoskeletal output that were based on tracking the desired trajectory.

The behavior of the high level controller was modeled by having access to a set of desired trajectories (i.e. joint angle and velocity profiles). To enrich the training set we used oscillatory trajectories of varying amplitude and frequency.

To simulate the control system efficiently, a number of C-codes were written taking full advantage of Matlab ${ }^{\circledR}$ and Simulink ${ }^{\circledR}$ (The Mathworks, Natick, MA, USA). The overall block diagram of the learning system is depicted in Fig. 4.

One of the major concerns in such a system is its stability. Therefore, we linearized the system about an equilibrium point so that the stability of the system in the neighborhood of the equilibrium point can be studied [21,30]. In addition, by assigning proper level of coactivation of the pair of muscles, we satisfied the stability requirements [31].

The reward function is evaluated according to equation 7 , where subscript e refers to the tracking error, which is difference between the desired and the actual response. The algorithm minimizes the reward function to track the reference trajectory with low activity.

$$
\begin{aligned}
\operatorname{Re}_{\text {ward }_{\text {Function }}} & =r_{1}\left|\theta_{e}\right|+r_{2}\left|\dot{\theta}_{e}\right|+r_{3}\left|l_{e}^{e}\right|+ \\
& r_{4}\left|l_{e}^{f}\right|+q_{1}\left|\alpha_{e x t}\right|+q_{2}\left|\alpha_{f x}\right|
\end{aligned}
$$

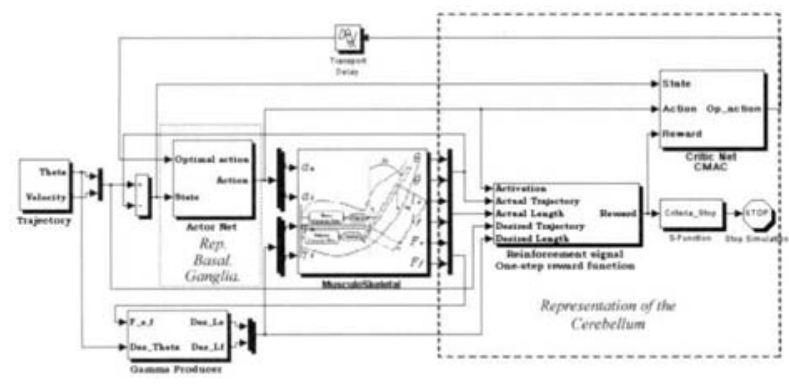

Fig.4 Block diagram of proposed learning system. The diagram consists of the Actor-Critic network, and musculoskeletal system.
Table I. RMS error of Trajectory and its Velocity during Training.

\begin{tabular}{|c|l|l|}
\hline Epoch & $\theta_{\mathrm{e}}(\mathrm{rad})$ & $\dot{\theta}_{\mathrm{e}}(\mathrm{rad} / \mathrm{sec})$ \\
\hline 1 & 1.187 & 2.174 \\
\hline 199 & 0.683 & 6.095 \\
\hline 280 & 0.023 & 0.042 \\
\hline 498 & 0.020 & 0.040 \\
\hline
\end{tabular}

\section{NUMERICAL EXAMPLE}

The learning process can be appreciated and quantified by looking at the joint position and velocity profiles achieved in Fig. 5 for training epochs 1, 199, 280 and 498 . The desired trajectory is an oscillatory movement with increasing frequency and constant amplitude. It is dear that main features of the desired trajectories have been learned by the $280^{\text {th }}$ epoch and further training only reduces the tracking error (Table 1).

The nature of learning process can also be depicted by showing the trend of reward function (Eq. 7) during simulation by mean and standard deviation in a number of epochs (Fig. 6). It is clear that initially the algorithm explores the decision space, but after the $280^{\text {th }}$ epoch the system becomes stable. Based on RMS error of performance adequate learning is achieved.

We illustrated the robustness of the learning and control algorithm by adding $2 \mathrm{Kg}$ load to the arm and simulating the oscillatory trajectory that was not used during the training algorithm. The model tracks the desired trajectory remarkably well, without the need for further training (Fig. 7.a). The RMS error for position and velocity were 0.024 radian, and 0.046 radian/sec, respectively.

Robustness and adaptive nature of algorithm were further examined by simulating tracking of a desired trajectory with increasing amplitude and frequency (Fig. 7.b). The RMS errors for position and velocity were $0.028 \mathrm{radian}, 0.055 \mathrm{radian} / \mathrm{sec}$, respectively.

The neural and sensory signals to achieve the desired trajectory shown in Fig. 7 are depicted in Fig. 8 . It is noted that the level of coactivation of extensor and flexor muscles require further work to account for energetic concerns. However, stable periodic movements have been tracked by properly activating the neural inputs. The alpha signals dominate the overall excitation signals to the muscle when compared to $R_{s p}$ and $R_{g t o}$ signals.

The forces generated by the muscles and exerted to the arm, also are shown in Fig. 9. 

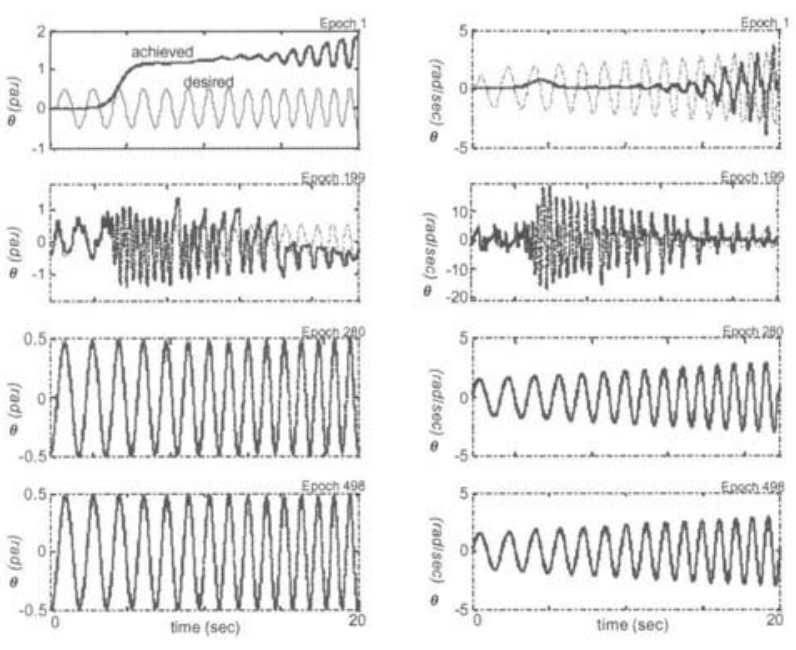

Fig.5 The achieved and desired trajectory and angular velocity at different epochs during learning process.

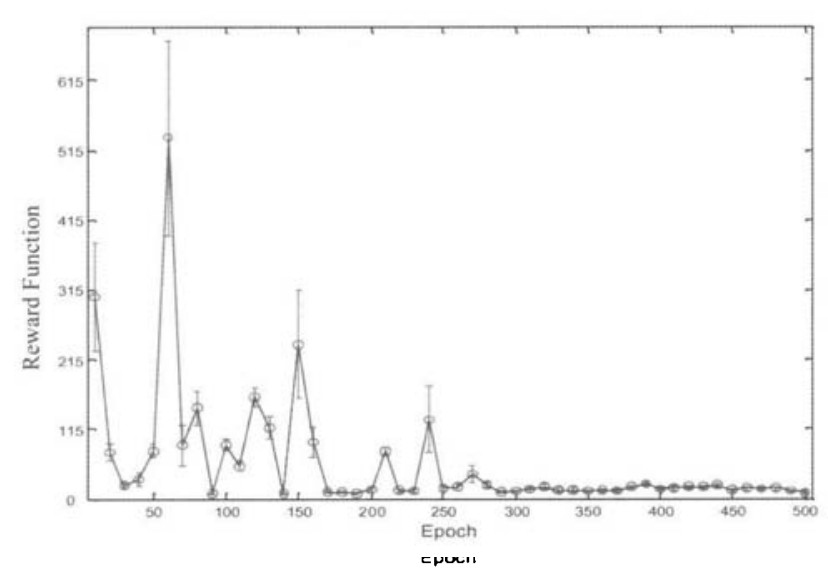

Fig.6 The exploration phase is characterized by fluctuating reward function, while after $280^{\text {th }}$ epoch the learning process has stabilized.

One of the limitations of current algorithm is the fact that a mathematical model was used to evaluate the desired gamma threshold based on joint angle and muscle force, while Kim and Hemami [16] proposed a separate neural network to achieve such a mapping. The following limitation may contribute to the exceptional robustness of the present algorithm. The relative role of different sensory and neural signals in the Reward allocation (Eq. 7) deserves further exploration. Multi-sensor data fusion, especially as we expand the model degrees of freedom and increase the complexity of the actuators system may be of concern.

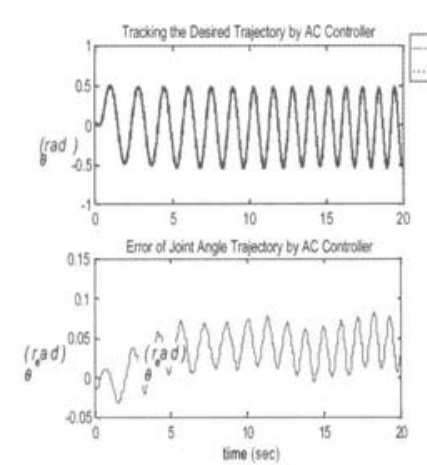

(a)

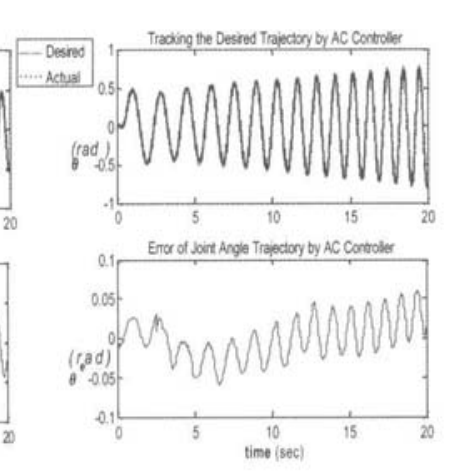

(b)
Fig. 7 (a) The effects of loading the arm with a point mass of $2 \mathrm{~kg}$ attached to its end, (b) the effects of increasing in movement amplitude.
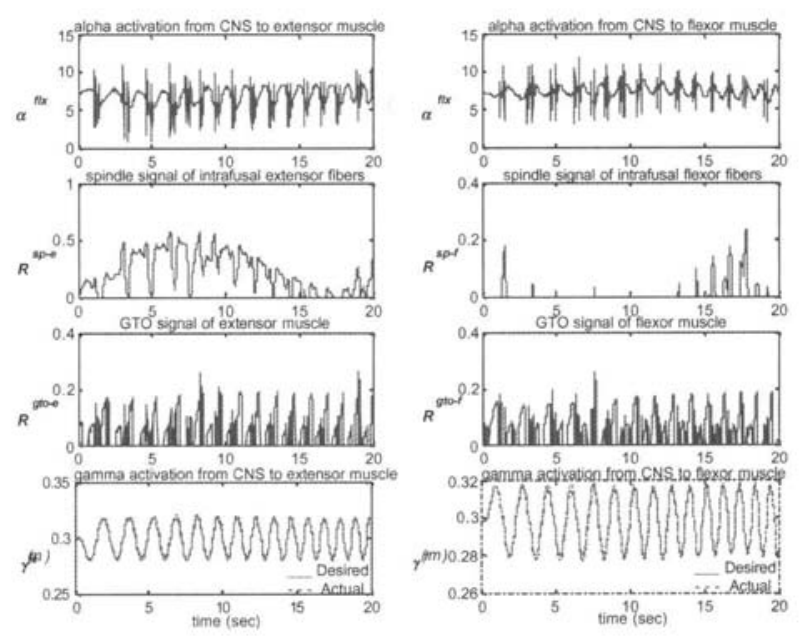

Fig.8 The spinal circuit signals for movement depicted in Fig 7.a.

\section{CONCLUSIONS}

The nature of sensorimotor information available in our control environment has guided our choice of reinforcement learning algorithm. Based on a trial-anderror approach to discover better controllers, the proposed learning method naturally optimizes our performance criteria over time. Houk et al. [14] have proposed the hypotheses that the basal ganglia might involve Actor-Critic learning which is a kind of reinforcement learning, while Critic here represented the behavior of cerebellum.

It is demonstrated that the Actor-Critic reinforcement learning method proposed here for the 

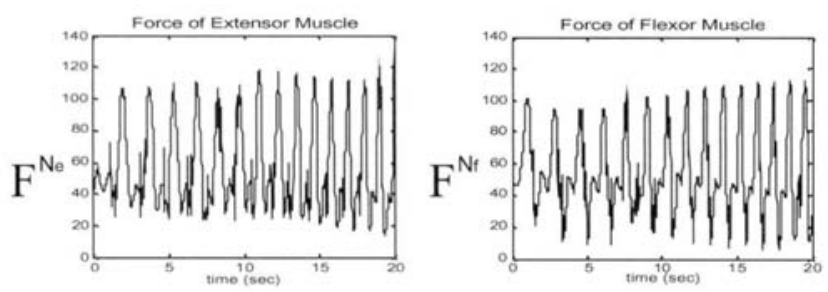

Fig.9 The required muscle forces for movement depicted in Fig 7.a.

mid-level CNS effectively learns and controls the desired maneuvers of the system after being trained with general patterns. In contrast to previous work [34], the Actor-Critic learning method proposed here generate physiological excitation signals to communicate with the musculoskeletal system. The proposed performance indices for our Actor-Critic learning method are more realistic than those using optimal control methods [8] and offline learning phase methods [16], resulting in a real time learning process.

In addition, we proposed the minimum excitation for both muscles and modeling error criterion as a reward definition. This enables the system obtain smooth movement with the low total muscle force which is reasonable for energy consumption or metabolic cost.

This nonlinear model was utilized to study repetitive movement of the system with spindle organ feedback and GTO feedback that are muscle sensors.

This work could be a step in the direction of a better understanding of the workings of the CNS in living systems by utilizing the capabilities of reinforcement learning and neural circuits to control highly nonlinear systems with multi-delays in the transmission paths of the system and redundant actuators.

For the next step, it may be desirable to improve this model to multiple links and multiple muscle system while paying more attention to essence of stability and muscle energetic.

\section{APPENDIX}

Definition of Variables and Numerical Parameters:

$\beta$ : stiffness constant for tendon $55000[\mathrm{~N} / \mathrm{m}]$

$\begin{array}{lc}m: \text { mass of the arm } & 1.9[\mathrm{Kg}] \\ g: \text { gravity constant } & 10\left[\mathrm{~m} / \mathrm{s}^{2}\right] \\ \tau & : \text { small positive fraction for the spindle }\end{array}$ feedback signal

0.2 $l_{c o}:$ initial length for contractile element

$v$ : small positive fraction for the Golgi tendon organ

0.1

$\xi$ : learning rate for back propagation algorithm 0.001

$\Delta$ : delay of feedback signal $\quad 40[\mathrm{~ms}]$

$\varepsilon$ : for $\varepsilon$-greedy policy 0.1

$F_{t h}:$ threshold force

$0[\mathrm{~N}]$

$\alpha$ : learning rate for SARSA algorithm 0.7

$H(s)$ : low-pass filter function of the tendon

$$
\frac{30}{s+30}
$$

$\gamma:$ discount factor

$l_{t o}$ : initial length for tendon $\quad 0.05[\mathrm{~m}]$

$N_{\text {hid }}$ : number of hidden layer for Actor

$c$ : passive elastic constant

controller

1

$b$ : viscous constant of the muscle $\quad 100$

$N_{\text {hid }}^{\prime}$ : number of node in hidden layer for Actor controller 14

$s_{1}, s_{2}$ : gain constant of spindle feedback

$D_{C M A C}$ : dimension of CMAC network 4

$z$ : gain constant of Golgi tendon organ

$n$ : number of intervals along each

dimension

$d$ : distance of the center of gravity from

$0.18[\mathrm{~m}]$

$N_{\text {tiling }}:$ number of tiling

20

$\Delta_{f}:$ time step used for simulation $0.01[\mathrm{~s}]$

$J$ : moment of inertia about the base

$0.075\left[\mathrm{Kg}-\mathrm{m}^{2}\right]$

$r_{i}(i=1 . .4)$ : coefficient of reward function are equal

100

$k:$ moment arm of the actuator

$q_{i}(i=1,2)$ : coefficient of reward

function are equal

$0.04[\mathrm{~m}]$

1

\section{REFERENCES}

1. Hemami $\mathrm{H}$ and Dinneen JA: A Marionette-Based Strategy for Stable Movement. IEEE Trans. Sys. Man Cyber 1993; 23: 502-511.

2. Hemami H: A State Space Model for Interconnected Rigid Bodies. IEEE Trans. Automatic Control 1982; 27: 376-382.

3. Kawato M, Furukawa $\mathrm{K}$ and Suzuki R: A Hierarchical Neural Network Model for Control and Learning of Voluntary Movement. Biol. Cyber 1987; 57: 169-185.

4. Kawato M, Uno $Y$, Isobe $M$ and Suzuki R: 
Hierarchical Neural Network Model for Voluntary Movement with Application to Robotics. IEEE Cont. Sys. Mag. 1988; 8: 8-16.

5. Pandy M, Anderson F and Hull D: A Parameter Optimization Approach for the Optimal Control of Large Scale, Musculoskeletal Systems. J. Biomech. 1992; 114: 450-460.

6. Zajac F: Muscle Coordination of Movement: A Perspective. J. Biomech. 1993; 26: 109-124.

7. Iqbal K, Hemami $\mathrm{H}$ and Simon S: Stability and Control of a Frontal Four-Link Biped System. IEEE Trans. Biomedical Eng. 1993; 40: 1007-1018.

8. Winters J: Concepts in Neuromuscular Modeling. Three Dimensional Analysis of Human Movement Eds. Allard P, Stokes I and Blanchi J, Champaign, IL: Human Kinetics, chap. 12, 1995; 257-292.

9. Izawa J, Kondo T and Ito K: Biological Robot Arm Motion through Reinforcement Learning. International Conference on Robotics \& Automation, Washington DC 2002; 3398-3403.

10. Izawa $J$, Kondo $T$ and Ito $K$ : Motor Learning using Reinforcement Learning with Neural Internal Model. Proceedings of IEEE International Conference on Robotics and Automation (ICRA'03), Taipei, Taiwan, 2003, (in press).

11. Ulusoy I, Parnianpour M, Berme N and Simon SR: A Neural Network System with Reinforcement Learning to Control a Dynamic Arm Model. Biomed Eng Appl Basis Comm 2001; 13: 117-123.

12. Althoefer L, Krekelberg B, Husmeire D and Seneviratne L: Reinforcement Learning in a rule based Navigator for Robot Manipulators. Neurocomputing 2001; 37: 51-70.

13. Martin P and Millan JR: Robot Arm Reaching through Neural Inversions and Reinforcement Learning. Robotics and Autonomous Systems $2000 ; 31: 227-246$.

14. Houk JC, Adams JL and Barto AG: A Model of How the Basal Ganglia Generate and Use Neural Signals that Predict Reinforcement. In Models of Information Processing in the Basal Ganglia. MIT Press, Cambridge, MA, USA, 1994; 249-270.

15. Jordan MI and Wolpert DM: Computational Motor Control. The Cognitive Neurosciences, MIT Press, 1999; 601-620.

16. Kim J and Hemami $\mathrm{H}$ : Coordinated Threedimensional Motion of the Head and Torso by dynamic Neural Networks. IEEE Trans. Sys. Man Cyber 1998; 653-666.

17. Kim J: Neural Excitation Training, and Control of Biological System. Ph. D. Dissertation, Department of Electrical Eng., The Ohio State Univ., Columbus, OH, 1995.

18. Kim J and Hemami H: Control of a One-Link Arm by Burst Signal Generators. Biol. Cyber 1995; 73: $37-47$.
19. Zajac F: Muscle and Tendon: Properties, Models, Scaling, and Application to Biomechanics and Motor Control. Critical Reviews in Biomedical Eng. 1989; 17: 359-411.

20. Gielen CC and Houk JC: A Model of the Motor Servo: Incorporating Nonlinear Spindle Receptor and Muscle Mechanical Properties. Biol. Cyber 1987; 57: 217-231

21. Dinneen JA and Hemami H: Stability and Movement of a One-Link Neuromusculoskeletal Sagittal Arm. IEEE Trans. Biomedical Eng. 1993; 40: $541-548$

22. Sutton RS and Barto AF: Reinforcement Learning: An Introduction. MIT Press, 1998.

23. Barto AF, Sutton $S$ and Anderson $C W$ : Neuronlike Adaptive Elements that Can Solve Difficult Systems. IEEE Trans. Sys. Man Cyber 1983; 13: 834-846.

24. Kretchmar RM: A Synthesis of Reinforcement Learning and Robust Control Theory. Ph. D. Dissertation, Department of Computer Eng., The Colorado State Univ., 2000.

25. Wolpert DM, Miall RC and Kawato M: Internal Models in the Cerebellum. Trends in Cognitive Sciences 1998; 2: 338-347.

26. Albus JS: A New Approach to Manipulator Control: The Cerebellar Model Articulation Control (CMAC). In Journal of Dynamic Systems, Measurement, and Control. Trans. ASME, Series G 1975; 97: 220-227.

27. Smith RL: Intelligent Motion Control with an Artificial Cerebellum. Ph. D. Dissertation, Department of Electrical Eng., University of Auckland, New Zealand, 1998.

28. Sutton RS: Generalization in Reinforcement Learning: Successful Examples using Sparse Coarse coding. In Advances in Neural Information Processing Systems 1996; 8: 10381044.

29. Tham CK: Reinforcement Learning of Multiple Tasks using a Hierarchical CMAC Architecture. Robotics and Autonomous Systems 1995; 15: 247 274.

30. Dariush B, Parnianpour $M$ and Hemami $H$ : Stability and a Control Strategy of a Multi-link Musculoskeletal Model with Applications in FES. IEEE Trans. on Biomedical Eng. 1998; 45: 3-14.

31. Jalics L, Parnianpour M, Barin K and Hemami H: Rhythmic Movement of a Pair of One-link Arms: Coordination by Intermitted Control. Computer Methods in Biomech. And Bio. Eng. 1999; 1: 2943. 